Published in final edited form as:

Lung Cancer. 2007 December ; 58(3): 403-410.

\title{
Sleep and quality of life in long-term lung cancer survivors
}

\author{
Nalaka S. Gooneratne, M.D., M.Sc. ${ }^{1,4}$, Grace E. Dean, Ph.D., R.N. ${ }^{2}$, Ann E. Rogers, Ph.D., R.N. \\ 3,4, J. Emeka Nkwuo, Ph.D.4 , James C. Coyne, Ph.D.5, and Larry R. Kaiser, M.D.6 \\ 1Division of Geriatric Medicine, University of Pennsylvania, Philadelphia, Pennsylvania \\ 2School of Nursing, State University of New York, Buffalo, New York \\ 3School of Nursing, University of Pennsylvania, Philadelphia, Pennsylvania \\ 4Center for Sleep and Respiratory Neurobiology and Division of Sleep Medicine, School of Medicine, \\ University of Pennsylvania, Philadelphia, Pennsylvania \\ 5Department of Psychology, University of Pennsylvania, Philadelphia, Pennsylvania \\ 6Department of Surgery, University of Pennsylvania, Philadelphia, Pennsylvania
}

\begin{abstract}
Background-Sleep problems are common in lung cancer survivors, yet little is known about the prevalence, determinants, and the effects on quality of life (QoL) of these sleep problems in longterm lung cancer survivors.

Methods-A case-control study design comparing 76 elderly lung cancer survivors (LCS, $>5$ years post diagnosis with mean survival time of 8 years $+/-2.1$ years) and 78 elderly non-cancer controls (NCC). Measurements included a standardized questionnaire for sleep (Pittsburgh Sleep Quality Index--PSQI), and analogue scales for dyspnea, pain, and other comorbid symptoms, as well as demographic factors and cancer history.
\end{abstract}

Results-Overall, $56.6 \%$ of LCS had poor sleep (PSQI global score $>5$ ) as compared to only $29.5 \%$ of NCC ( $\mathrm{p}<0.001$ ), and $49.2 \%$ of LCS who did not have sleep difficulties prior to their lung cancer diagnosis ultimately developed them. There was also evidence of significant impairments in sleep efficiency in LCS $(78.3 \%)$ relative to $\mathrm{NCC}(89.6 \%$, p $<0.001)$, predominantly due to increased nocturnal awakenings. A single-item analogue scale for sleep quality was not as effective in identifying sleep problems as more specific questions about sleep duration and sleep efficiency. Poor sleep quality was significantly correlated with impairments in quality of life, even when controlling for other factors, such as dyspnea.

Conclusions-Even 8 years after diagnosis, LCS continue to have significant sleep difficulties. By asking specific questions about sleep medication use, nocturnal awakenings and sleep efficiency,

Please address all correspondence to: Nalaka S. Gooneratne, M.D., M.S.C.E., Ralston House, Rm. 220, 3615 Chestnut St., Philadelphia, PA 19104, Telephone: 215-349-5938 / Fax: 215-573-8684, e-mail: ngoonera@mail.med.upenn.edu.

Abstract presented at the 2005 Annual Meeting of the Associated Professional Sleep Societies, Denver, Colorado (June 2005)

Informed Consent: This study was approved by the University of Pennsylvania Institutional Review Board \#8 as a low-risk exempt protocol, ID \# 708663. It was approved for a HIPAA authorization waiver. The protocol was also evaluated and approved by the University of Pennsylvania Cancer Center (UPCC) Clinical Trials Scientific Review and Monitoring Committee (CTSRMC), protocol ID \# 06503. Verbal informed consent was obtained on all subjects participating in the study.

Conflict of interest statement No authors have conflicts of interest related to this publication.

Publisher's Disclaimer: This is a PDF file of an unedited manuscript that has been accepted for publication. As a service to our customers we are providing this early version of the manuscript. The manuscript will undergo copyediting, typesetting, and review of the resulting proof before it is published in its final citable form. Please note that during the production process errors may be discovered which could affect the content, and all legal disclaimers that apply to the journal pertain. 
health care providers can identify sleep problems that could be treated and potentially improve the quality of life of their patients.

\section{Keywords}

Lung cancer; sleep disorders; cancer survivor; aged; quality of life; long-term survival

\section{Introduction}

Improvements in the diagnosis and treatment of lung cancer have resulted in increased opportunities for long-term survival [1,2]. Indeed, approximately 26,000 Americans per year with lung cancer will go on to survive for more than five years from the time of initial diagnosis [3]. This has led to a growing interest in addressing issues faced by these long-term survivors [4]. Sleep disorders are particularly common in lung cancer survivors, with $31 \%$ to $52 \%$ reporting insomnia symptoms [5-8]; these prevalence rates were found to be markedly higher than controls $[7,9]$.

Furthermore, these sleep disorders lead to pronounced symptom distress: Sarna and colleagues gathered symptom distress data on female lung cancer patients an average of 1.8 years after treatment and found that insomnia was a major concern; it ranked as the third highest cause of distress after fatigue and pain [8]. Of note, research in non-cancer patients has shown that insomnia is associated with significant sequelae [10], such as an increased risk of accidents [11], depression [12] and impaired quality of life [13]. Even in relatively healthy adults, while controlling for baseline medical status and using objective criteria for insomnia, the presence of insomnia led to a 1.93 times greater risk of death over 4-19 years of follow-up [14]. Despite these concerns, insomnia is often undetected and under-treated when detected [15,16], with only $16.6 \%$ of patients in one study having spoken to their physician about their sleep problems [17].

Past studies that have examined sleep disturbances in lung cancer survivors have been helpful in providing estimates of prevalence and potential risk factors, but there are several limitations in this literature. First, many of these studies have had only a sparse assessment of sleep disturbances, relying on the symptom complaint of difficulty initiating or maintaining sleep [7,8], and have not inquired about other sleep disorders or used standardized clinical or research diagnostic criteria for insomnia as a syndrome $[18,19]$. This can lead to high estimates for the prevalence of the insomnia symptom that exceed the prevalence of insomnia as a syndrome/ disease [20]. The one prior study that used more rigorous criteria was limited by a small sample size [21]. Second, these studies have sampled patients who, on average, were not long-term ( $>5$ years) lung cancer survivors $[7,17,22]$. Third, many of these studies have included a broad range of cancer types, not just lung cancer [7,21]. The observation that lung cancer survivors have some of the highest rates of insomnia when compared to other cancer survivors suggests that there may be unique factors related to lung cancer survivorship that influence sleep [7, 23]. Candidate factors that may be associated with sleep disruption include pain [6] and depression [24]. Furthermore, lung cancer survivors may also face additional problems from increased rates of dyspnea [25].

In order to address these issues, we conducted the first case-control study, to our knowledge, of sleep disturbances in an elderly lung cancer survivor population that had survived for more than five years since the initial diagnosis. We used a validated standardized sleep disturbance questionnaire, and included a control population of elderly patients without a cancer history. Our aim was to provide a more detailed description of sleep disturbances in lung cancer survivors by determining prevalence rates and risk factors for these sleep problems, with a particular focus on insomnia symptoms. 


\section{Methods}

\section{Participants}

Subjects were drawn from the clinic records of the Hospital of the University of Pennsylvania, an academic teaching university which has a catchment area extending across eastern Pennsylvania, Delaware and southern New Jersey. We limited our study to subjects over the age of 60. Lung cancer is often a disease of older adults [3], however, past studies tend to include subjects across age groups. Given that there are significant age-related effects on a variety of sleep disorders [26,27], we decided to only enroll subjects over the age of 60. Subjects with lung cancer were drawn from the Hospital of the University of Pennsylvania Thoracic Oncology Clinic. To be eligible, subjects had to be at least five years post-diagnosis of their lung cancer (long-term lung cancer survivors, LCS). Non-cancer controls (NCC) were also drawn from the Hospital of the University of Pennsylvania clinic population and had no history of cancer. The Hospital of the University of Pennsylvania maintains an elder outreach and education program, Penn Partner's in Healthy Living. Patients who attended the out-patient clinics of the hospital were invited to enroll in the program. An additional function of the Penn Partners in Health Living program is to assist in notifying elders of ongoing research projects and thereby serve as a research study recruitment tool, as in the case with this study. NCC over the age of 60 were randomly selected from this database.

\section{Instruments}

All sleep data was obtained from the Pittsburgh Sleep Quality Index (PSQI) [28]. It is a selfrated questionnaire that consists of 11 questions, some of which contain sub-questions so that there are a total of 20 questions. Questions include bedtime, wake time, and time spent sleeping. These were used to calculate sleep efficiency: (wake time - bedtime) / time spent sleeping). A range of sleep disturbances are also assessed in the PSQI, including sleep quality, insomnia, sleep apnea and limb movement disorders, as well as daytime consequences of sleep and sleep medication use. The PSQI has been used in clinical and research settings to distinguish good sleepers (global score < $=5$ ) and poor sleepers $(>5)$, and is responsive to changes in insomnia [29]. Good measures of internal homogeneity, consistency (test-retest reliability), and validity have been obtained on psychometric testing [28,30]. It has also been used in cancer research [31] and in a telephone interview format where it correlated well with narrative comments from open-ended interviews [32].

Subjects were also asked about their lung cancer history, demographic information and other symptoms related to potential comorbid conditions associated with sleep disturbances. The specific symptoms assessed were pain severity, depression and dyspnea because these have been identified in past studies as some of the most common causes of distress in lung cancer patients $[8,33]$. The symptoms were measured using a 0-10 interval scale as this has been found to represent a straightforward and accurate assessment modality [34]. Overall perception of quality of life was also addressed using the following question: "How good is your quality of life?" The response options also ranged from 0 ("Extremely poor") to 10 ("Excellent"). A single-item quality of life assessment has been found to have good correlation with more detailed instruments [35,36].

All subjects were contacted via telephone. The PSQI and other rating instruments were administered during a telephone interview conducted by the study investigators.

\section{Statistical Analysis}

The primary analysis involved several stages. First, estimates of the prevalence of various sleep disturbances among both LCS and NCC were determined, along with 95\% confidence intervals. The PSQI global score was also calculated in these two groups. Because we are also 
interested in the effects of sleep on quality of life in lung cancer survivors, we then did a further analysis involving lung cancer survivors only. For this analysis, poor sleepers were defined as lung cancer survivors with a PSQI global score greater than five, and good sleepers were lung cancer survivors with a PSQI global score less than or equal to five. Univariate analyses were then conducted to examine the relationship between their QoL rating and various demographic and comorbid symptoms. Factors that were significant were then studied for evidence of interaction effects using a correlation matrix and further explored with bivariate regression models. Items that were significant at an alpha $<0.05$ were then considered for inclusion in a multiple linear regression model to identify determinants of QoL. All statistical tests presented in this analysis are two-tailed, with statistical significance defined as $p<0.05$. The statistical analysis was performed using SAS v9.1 (SAS Institute Inc., Cary, North Carolina).

\section{Results}

The response rate to our survey was $61.8 \%$. Demographic characteristics of our study population are presented in Table 1 . There were no statistically significant differences between lung cancer survivors (LCS) and non-cancer controls (NCC) for any of these demographic parameters. The mean time duration of survival for our LCS was 8.0 years $(+/-2.1$, range: 5-18 years). The majority of LCS were treated with surgical therapy only $(89.5 \%)$, with only a minority receiving additional chemotherapy $(2.6 \%)$, radiation therapy $(1.3 \%)$ or combination therapy $(6.6 \%)$. There were no differences by race or gender in the treatments they received for their lung cancer.

\section{Sleep Parameters}

There were significant differences in self-reported sleep parameters between LCS versus NCC. The LCS reported spending more hours in bed relative to NCC $(8.4+/-1.3$ hours vs. $7.9+/$ 1.2 hours, $\mathrm{t}=2.59, \mathrm{p}=0.01)$, but less time asleep in bed $(6.5+/-1.4$ hours vs. $7.0+/-1.4$ hours, $\mathrm{t}=2.27, \mathrm{p}=0.02)$. This resulted in statistically significant differences in self-reported sleep efficiency, with LCS having a sleep efficiency of $78.3 \%+/-15.2 \%$ and NCC having a sleep efficiency of $89.6 \%+/-15.8 \%(\mathrm{t}=4.53, \mathrm{p}<0.001)$. However, differences in sleep efficiency were not due to changes in self-reported sleep latency (i.e., time it takes to fall asleep), which was similar between groups (LCS $24.0+/-24.4$ min vs. NCC $19.9+/-19.6 \mathrm{~min}, \mathrm{t}=1.17, \mathrm{p}=0.24$ ). Thus, the reduced sleep efficiency most likely reflects increased rates of wakefulness after sleep onset. Indeed, when specifically asked if they "Cannot get to sleep within 30 minutes", there was no difference between the groups (chi-square $=2.88, \mathrm{p}=0.4$ ), but when asked if they "Wake-up in the middle of the night or early morning, $40.8 \%$ of LCS noted that this happened three or more times a week relative to only $21.8 \%$ of NCC (chi-square $=10.75, \mathrm{p}=0.01$ ).

The average bedtime and wake time is presented in Figure 1. It demonstrates evidence of a tendency towards more time in bed for LCS with a later wake time than NCC: LCS went to bed an average of 6 minutes earlier than NCC $(\mathrm{t}=0.46, \mathrm{p}=0.6)$, and LCS got up an average of 26 minutes later than $\mathrm{NCC}(\mathrm{t}=2.03, \mathrm{p}=0.04)$.

When asked about common causes of insomnia that interfered with their sleep once or more during the past month (Table 2), NCC were significantly more likely to mention pain as a factor. Other causes of poor sleep that occurred more frequently in LCS than NCC but did not reach statistical significance were dyspnea $(\mathrm{p}=0.06)$ and nocturia $(\mathrm{p}=0.07)$.

The presence of symptoms suggestive of other sleep disorders aside from insomnia was also assessed by asking subjects if they had ever been told they had additional sleep symptoms. NCC were more likely to report that they snored than LCS $(28.2 \%$ of NCC vs. $14.5 \%$ of LCS, chi-square $=4.3, \mathrm{p}=0.04$ ). The occurrence of symptoms of other sleep disorders were not found 
to differ significantly between groups, with no difference in history of witnessed pauses in breathing (a possible sign of sleep apnea), restless legs/tremors, and nocturnal confusion.

When asked to rate their overall sleep quality, 25.3\% of LCS described their sleep as very bad or fairly bad compared to only $11.7 \%$ of NCC (chi-square $=4.7, \mathrm{p}=0.03$ ). This is corroborated by the finding that they were significantly more likely to use sleeping medications (chisquare $=8.9, \mathrm{p}=0.03$ ), with $19.7 \%$ of LCS using them three or more times a week as compared to only $11.5 \%$ of NCC. Overall, $31.6 \%$ of LCS had used a sleeping aid at some point during the past month as compared to only $15.4 \%$ of NCC. Despite these differences, when asked how much their sleep problems bothered them, LCS and NCC reported similar levels of distress $($ LCS $2.7+/-3.0$ vs $2.3+/-2.6,0=$ not at all, $10=$ a great deal, $\mathrm{t}=0.68, \mathrm{p}=0.5)$.

\section{Pittsburgh Sleep Quality Index}

We next computed PSQI global (Figure 2) scores for LCS and NCC. Statistically significant differences were noted between the PSQI global score in LCS and NCC $(6.3+/-4.1$ in LCS vs. $4.5+/-3.1$ in $\mathrm{NCC}, \mathrm{t}=3.15, \mathrm{p}=0.002$ ). Values above five on the PSQI are considered indicative of poor sleep quality, and according to this criteria, LCS were more likely to have poor sleep than NCC [28]. Indeed, 56.6\% (95\% C.I.: 45.2\% - 70.0\%) of LCS had PSQI scores greater than five as compared to only $29.5 \%$ (95\% C.I.: $19.1 \%-39.8 \%$ ) of NCC (chi-sq 11.5 , $\mathrm{p}=0.0007)$.

To determine the influence of pre-cancer insomnia on current insomnia, we asked lung cancer survivors if they had sleep problems prior to their cancer diagnosis. Of LCS who currently have poor sleep (PSQI>5), 28.6\% reported having had sleep problems in the past prior to their cancer diagnosis. However, only $9.1 \%$ of LCS who currently report good sleep (PSQI $<=5)$ noted that they had sleep problems prior to their cancer diagnosis (chi-square $=4.4, \mathrm{p}=0.04$ ). In addition, $49.2 \%$ (95\% C.I.: $36.2 \%$ to $62.1 \%$ ) of LCS who did not have sleep difficulties prior to their lung cancer diagnosis would go on to develop these problems over the average 8 year survival time of our LCS population.

\section{Other Symptoms and Quality of Life}

When asked about other common symptoms, LCS and NCC had similar ratings for depression symptoms ( $\mathrm{t}=1.11, \mathrm{p}=0.3$ ), but NCC tended to report more problems due to pain (LCS $2.1+$-/3.1 vs. NCC $3.0+/-3.2,0=$ no problem, $10=$ severe problem, $\mathrm{t}=1.83$, $\mathrm{p}=0.07)$. Dyspnea was significantly more likely to cause a problem for LCS than NCC (LCS $2.6+/-3.4$ vs. NCC 1.1 $+/-2.5,0=$ no problem, $10=$ severe problem, $\mathrm{t}=3.08, \mathrm{p}=0.003$ ). However, when asked to rate their overall quality of life, LCS and NCC had similar self-ratings that were both fairly positive (LCS $8.0+/-2.0$ vs. NCC $8.4+/-1.6,0=$ extremely poor, $10=$ excellent, $\mathrm{t}=1.36, \mathrm{p}=0.18$ ).

\section{Association Between Poor Sleep and QoL}

We next focused our analysis on LCS with poor sleep by PSQI criteria $(>5)$ and compared them to LCS with good sleep (PSQI $<=5$ ). We found significant associations between poor sleep and QoL, with LCS with poor sleep having a QoL rating that was significantly lower than LCS with good sleep quality $(7.3+/-2.3$ vs. $8.8+/-1.1, \mathrm{t}=3.63$, $\mathrm{p}=0.0005)$. NCC with poor sleep also had worse QoL than NCC with good sleep (QoL in NCC with poor sleep: 7.6 $+/-1.8$ vs. QoL in NCC with good sleep: $8.7+/-1.3, \mathrm{t}=2.85, \mathrm{p}=0.006)$. The difference in $\mathrm{QoL}$ as a function of sleep quality was $1.6+/-1.9$ for LCS and $1.1+/-1.5$ for NCC; however, this was not statistically significant $(\mathrm{p}=0.15)$. 


\section{Determinants of QoL in LCS}

While the above analysis demonstrates evidence to suggest that poor sleep is common in lung cancer survivors and that it has an impact on QoL, the overall significance of this poor sleep within the context of other symptoms these individuals may experience is not clear. To further elucidate this, we performed a multivariate regression analysis of QoL determinants in the lung cancer survivors only $(\mathrm{n}=76)$. As an initial step in our analysis, we conducted univariate analysis between several potential QoL determinants and QoL itself (Table 3).

PSQI global score (sleep quality) and dyspnea were the only factors with significant associations with QoL. Given the potential relationship between sleep quality and dyspnea, we then examined the data for evidence of an interaction between these two conditions by constructing a regression model that included an interaction term: no significant interaction was noted in that model, with the interaction term having a parameter coefficient $t$-value $=0.34$, $\mathrm{p}=0.7$.

We used the above findings to construct a linear regression model for QoL that included the significant factors from the univariate analysis. The model was statistically significant $(\mathrm{F}=5.2$, $\mathrm{p}=0.008$ ); the parameter coefficients are presented in Table 3. For each one point increase in the PSQI global score, there was a 0.11 decrease in the QoL (as defined on an analogue scale of 0-10), and for each 1 point increase in dyspnea severity (as defined also on an analogue scale of 0-10), there was a 0.14 decrease in the QoL. However, the adjusted r-square of the model was low at 0.10 . Model diagnostic assessment included collinearity diagnostics that showed variance inflation factors that were all less than ten, and acceptable eigenvalues of the correlation matrix.

\section{Discussion}

This study is one of the first to examine sleep patterns in long-term ( $>5$ years) lung cancer survivors and compare them to non-cancer controls. Our findings were notable for the fact that the sleep efficiency of lung cancer survivors was markedly lower than the sleep efficiency of non-cancer controls, thus suggesting increased rates of sleep disruption in LCS. For the majority of lung cancer survivors, these sleep problems did not predate their cancer diagnosis. A review of their sleep patterns suggested evidence of a delay in wake times as well, while bed times were similar. These sleep difficulties were associated with significant impairments in QoL in our lung cancer survivors, even when controlling for dyspnea. Equally troubling, these lung cancer survivors had higher rates of sleep medication use relative to non-cancer controls.

Research in cancer survivors in general has shown that sleep difficulties are a common concern, with 36.9-58.7\% of cancer survivors an average of one year after diagnosis reporting sleep symptoms [37]. Lung cancer patients, when compared to other patients with the three most common cancers (breast, colorectal and prostate cancer), had the highest percentage of these sleep problems (58.7\%) [37]. Despite this high prevalence, relatively few studies have focused on sleep in lung cancer patients, and most of these have had subject populations that were in the peri-treatment period $[21,33]$, or were short-term $(<5$ year) survivors $[7,8,17]$.

These studies have found that there are marked abnormalities in sleep in lung cancer patients. Objective measurement of sleep using polysomnography, the gold standard objective measure of sleep architecture, has found that the sleep efficiency of lung cancer patients was lower (79.5\%) than normal sleepers (88.4\%) or breast cancer patients (84.8\%) [21]. Davidson and colleagues studied patients with various types of cancer an average of 34 months from the time of diagnosis. They noted that $48.2 \%$ of patients developed their sleep problems 6 months before to 18 months after their diagnosis [7]; this suggest that many sleep difficulties are present from the peri-treatment period onwards, a finding that we observed as well. Recall bias can be a 
concern with these types of retrospective studies, thus our finding may, to a certain extent, be an overestimate.

The insomnia complaints noted by Davidson and colleagues in their study of predominantly older patients (mean age of 64.9 years) with various types of cancer tended primarily to be difficulty maintaining sleep (76\%), followed by difficulty falling asleep (44\%) and early morning awakenings (33\%) [7]. Our study observed a similar pattern, with the majority of patients reporting difficulty staying asleep. Interestingly, a recent meta-analysis of sleep patterns throughout the lifespan also concluded that with advancing age, the main cause of reduced sleep efficiency was an increase in wakefulness after sleep onset; sleep latency tended to remain stable throughout the lifespan and contributed relatively little to the age-associated decrease in sleep efficiency [27].

Lung cancer survivors may have high rates of sleep difficulties because they may have more comorbid conditions than most other cancer survivors [37]; these comorbidities are clearly a key risk factor for sleep disruption [38]. In part, the higher rate of comorbidity may occur because the majority of lung cancer patients were exposed to tobacco and thus there is a higher than average presence of tobacco-related illnesses in lung cancer patients [39]. For example, $52.1 \%$ of long-term ( $>5$ year) lung cancer survivors have moderate to severe pulmonary disease as defined by an FEV1<70\% [39]. When considering emphysema in particular, there is clear evidence that insomnia is common in these patients [25]. Indeed, one study that performed factor analysis of symptom distress and physical complaints noted that insomnia had the highest factor loading with the respiratory distress component [33]. Other conditions that have been associated with insomnia in cancer patients include psychiatric conditions such as depression or anxiety, and increased levels of stress and pain [7,23]. These conditions are most commonly associated with insomnia in patients who have been recently diagnosed or are undergoing treatment. It may be that we did not find strong associations between these factors and sleep disruption in our long-term lung cancer survivor population because they were an average of eight years post-diagnosis.

One of the most interesting findings from our study was the observation that while a singleitem analogue scale screen for distress from insomnia did show increased distress in lung cancer survivors relative to non-cancer controls, it was not a statistically significant difference. In contrast, the more comprehensive assessment provided by the Pittsburgh Sleep Quality Index showed robust differences. Several studies, including one that attempted to develop a singleitem screen for insomnia [40] as well as research with advanced lung cancer patients [22], have found that a single-item assessment of sleep quality is of limited utility. Silberfarb and colleagues in their study of breast cancer, lung cancer and normal patients, observed that lung cancer patients tended to markedly underreport their sleep problems when the authors compared the objective sleep findings from polysomnography data to the subjective interview and sleep analogue scale responses [21]. Breast cancer patients, on the other hand, were noted to have objective polysomnography sleep findings that were fairly similar to normals despite a high degree of subjective sleep disruption complaints [21]. The authors postulated that this unique underreporting of sleep difficulties in lung cancer patients may be due to denial or lack of awareness of their own internal mood states [21]. It was most likely not due to gender differences as half of their lung cancer patients were female as well [21].

Another possible explanation for the tendency to underreport sleep complaints on a single-item question is that the lung cancer survivors did indeed feel less distress from their sleep problems. We observed that evidence of increased sleep difficulties only arose on more detailed questions that asked about specific aspects of sleep, such as sleep efficiency (time in bed divided by time asleep) and sleep medication use. This reduced perception of insomnia-related distress may have been mediated by several factors. One of these mediating factors may be a response shift: 
these lung cancer survivors have become habituated to some of their symptoms, a phenomenon also noted amongst other long-term cancer survivor groups such as ovarian cancer survivors [41]. For example, despite the fact that $53.5 \%$ of these long-term ovarian cancer survivors reported pain or other discomfort, $89.0 \%$ described their health as good or excellent [41]. Amongst long-term lung cancer survivors, $50 \%$ felt that their lung cancer had a positive effect on their life, and $71 \%$ described themselves as hopeful [39]. When comparing quality of life, the Short Form 36 Physical Component score of these lung cancer survivors was lower than published scores for chronic lung disease patients, yet the Mental Component score for the lung cancer survivors was higher [39]. An additional factor mediating the reduced perception of distress from sleep problems could be the higher rate of sleep medication use we noted in our lung cancer survivors relative to non-cancer controls; one can postulate that these patients may be receiving a treatment benefit from the sleep medications that reduced their perception of distress from insomnia.

Another important question to consider is the role of sleep disruption in terms of the broader context of QoL. We noted that a comprehensive assessment of sleep disruption by the Pittsburgh Sleep Quality Index revealed strong associations between sleep difficulties and quality of life. The only other factor we found to be significant for QoL was dyspnea. While the standardized parameter coefficients in our multivariate regression model were similar for these two terms, it is important to emphasize that they were scored differently; a one point change on an analogue scale is not necessarily the same as a one point change in the Pittsburgh Sleep Quality Index global score. We also did not find a significant link between QoL and depression or pain, but this may have been due to the relatively low levels of these complaints. Silberfarb and colleagues also noted low levels of depression in their study of lung cancer patients and sleep [21]. It is also important to interpret our multivariate regression model findings cautiously because, as with other research exploring QoL [42], our model explained a relatively small percentage of the variation in QoL. Nevertheless, these findings do suggest that sleep factors are an important aspect of QoL and corroborate findings noted in other research in non-cancer populations [43].

Despite the effects of sleep difficulties on quality of life, these sleep disorders are often not evaluated or treated [17]. In part this is because health care providers do not inquire about sleep, but the patients themselves do not report it as well in many cases [17]. Open-ended questions revealed that many patients variously felt their health care provider could do little to treat the condition, were reluctant to take sedative-hypnotics, believed it was something they just had to deal with, or felt that it was not important within the broader context of their cancer [17].

Our study has limitations. Most of our subjects had surgery and few had chemotherapy or radiation therapy; current treatment recommendations often call for these as neo-adjuvant treatments. The more frequent use of these neo-adjuvant methods in current lung cancer treatment paradigms may increase the prevalence of insomnia. In addition, our study did not control for factors such as smoking. This can worsen sleep quality, but most likely has little effect on QoL as other research has found that smoking was not predictive of QoL [39]. We also did not measure caffeine intake, or medications that have been implicated as exacerbating insomnia. Of note, in one large study of cancer survivors in general, lifestyle factors such as caffeine use, alcohol and environment were not found to be major contributors to insomnia [7]. Nevertheless, measurement of these factors would be worthwhile in future studies. Our study also measured only a limited range of comorbid symptoms and relied on self-report assessments; more comprehensive instruments may reveal additional associations. Our data on sleep, while more detailed, was also derived from self-report measures. While the diagnosis of insomnia is primarily a clinical one based on subjective perceptions [19], objective data on sleep patterns would be insightful. Future research should include a more comprehensive 
assessment of the various risk factors as well as objective measures of sleep in an attempt to more accurately define the relationship of sleep disorders with comorbid diseases and quality of life that we have noted.

In conclusion, our study demonstrates that even in long-term lung cancer survivors, sleep problems are significantly more frequent than in non-cancer controls of a similar age. Many of our lung cancer survivors developed these sleep difficulties after their lung cancer diagnosis. The etiology of these sleep problems also appeared to be different from the non-cancer controls with dyspnea playing a greater role in disrupting the sleep of lung cancer survivors. These sleep difficulties had a significant and concerning association with impaired QoL in our lung cancer survivors. They were also associated with increased rates of sedative-hypnotic use. New research showing the potential benefits of cognitive behavioral therapy $[44,45]$ as well as improvements in pharmacotherapy [46,47] may offer increasing effectiveness in treating these patients. Asking lung cancer survivors detailed and concrete questions about their sleep, such as how often they have nocturnal awakenings or how long they are awake each night, is thus a worthwhile and important aspect of their care.

\section{Acknowledgements}

The investigators would like to thank Marion Whitlock, Elizabeth Roben and Lea Riedi for their assistance with this project.

Supported by NIH contracts NIA K23 AG01021, NCRR M01 RR00040 and the Veterans Affairs Competitive Pilot Project Fund, VISN 4.

\section{References}

1. Okamoto T, Maruyama R, Shoji F, Asoh H, Ikeda J, Miyamoto T, et al. Long-term survivors in stage IV non-small cell lung cancer. Lung Cancer 2005;47(1):85-91. [PubMed: 15603858]

2. Brock MV, Hooker CM, Syphard JE, Westra W, Xu L, Alberg AJ, et al. Surgical resection of limited disease small cell lung cancer in the new era of platinum chemotherapy: Its time has come. J Thorac Cardiovasc Surg 2005;129(1):64-72. [PubMed: 15632826]

3. Jemal A, Tiwari RC, Murray T, Ghafoor A, Samuels A, Ward E, et al. Cancer statistics, 2004. CA Cancer J Clin 2004;54(1):8-29. [PubMed: 14974761]

4. Rowland JH, Aziz N, Tesauro G, Feuer EJ. The changing face of cancer survivorship. Semin Oncol Nurs 2001;17(4):236-40. [PubMed: 11764706]

5. Ginsburg ML, Quirt C, Ginsburg AD, MacKillop WJ. Psychiatric illness and psychosocial concerns of patients with newly diagnosed lung cancer. Cmaj 1995;152(5):701-8. [PubMed: 7882232]

6. Portenoy RK, Miransky J, Thaler HT, Hornung J, Bianchi C, Cibas-Kong I, et al. Pain in ambulatory patients with lung or colon cancer. Prevalence, characteristics, and effect. Cancer 1992;70(6):161624. [PubMed: 1516015]

7. Davidson JR, MacLean AW, Brundage MD, Schulze K. Sleep disturbance in cancer patients. Soc Sci Med 2002;54(9):1309-21. [PubMed: 12058848]

8. Sarna L. Correlates of symptom distress in women with lung cancer. Cancer Pract 1993;1(1):21-8. [PubMed: 8324526]

9. Malone M, Harris AL, Luscombe DK. Assessment of the impact of cancer on work, recreation, home management and sleep using a general health status measure. J R Soc Med 1994;87(7):386-9. [PubMed: 8046723]

10. Taylor DJ, Lichstein KL, Durrence HH. Insomnia as a health risk factor. Behav Sleep Med 2003;1 (4):227-47. [PubMed: 15600216]

11. Gislason T, Reynisdottir H, Kristbjarnarson H, Benediktsdottir B. Sleep habits and sleep disturbances among the elderly--an epidemiological survey. J Intern Med 1993;234(1):31-9. [PubMed: 8326287]

12. Weissman MM, Greenwald S, Nino-Murcia G, Dement WC. The morbidity of insomnia uncomplicated by psychiatric disorders. Gen Hosp Psychiatry 1997;19(4):245-50. [PubMed: 9327253] 
13. Schubert CR, Cruickshanks KJ, Dalton DS, Klein BE, Klein R, Nondahl DM. Prevalence of sleep problems and quality of life in an older population. Sleep 2002;25(8):889-93. [PubMed: 12489896]

14. Dew MA, Hoch CC, Buysse DJ, Monk TH, Begley AE, Houck PR, et al. Healthy older adults' sleep predicts all-cause mortality at 4 to 19 years of follow-up. Psychosom Med 2003;65(1):63-73. [PubMed: 12554816]

15. Reynolds CF 3rd, Buysse DJ, Kupfer DJ. Treating insomnia in older adults: taking a long-term view. JAMA 1999;281(11):1034-5. [PubMed: 10086440]

16. Haponik EF. Sleep disturbances of older persons: physicians' attitudes. Sleep 1992;15(2):168-72. [PubMed: 1579792]

17. Engstrom CA, Strohl RA, Rose L, Lewandowski L, Stefanek ME. Sleep alterations in cancer patients. Cancer Nurs 1999;22(2):143-8. [PubMed: 10217030]

18. Edinger JD, Bonnet MH, Bootzin RR, Doghramji K, Dorsey CM, Espie CA, et al. Derivation of research diagnostic criteria for insomnia: report of an American Academy of Sleep Medicine Work Group. Sleep 2004;27(8):1567-96. [PubMed: 15683149]

19. American Psychiatric Association. Diagnostic and statistical manual of mental disorders : DSM-IVTR. 4th. Washington DC: American Psychiatric Association; 2000.

20. Billiard M, Bentley A. Is insomnia best categorized as a symptom or a disease? Sleep Med 2004;5:S35-40. [PubMed: 15301996]

21. Silberfarb PM, Hauri PJ, Oxman TE, Schnurr P. Assessment of sleep in patients with lung cancer and breast cancer. J Clin Oncol 1993;11(5):997-1004. [PubMed: 8487063]

22. Vena C, Parker K, Allen R, Bliwise D, Jain S, Kimble L. Sleep-wake disturbances and quality of life in patients with advanced lung cancer. Oncol Nurs Forum 2006;33(4):761-9. [PubMed: 16858458]

23. Savard J, Morin CM. Insomnia in the context of cancer: a review of a neglected problem. J Clin Oncol 2001;19(3):895-908. [PubMed: 11157043]

24. Savard J, Simard S, Blanchet J, Ivers H, Morin CM. Prevalence, clinical characteristics, and risk factors for insomnia in the context of breast cancer. Sleep 2001;24(5):583-90. [PubMed: 11480655]

25. George CF, Bayliff CD. Management of insomnia in patients with chronic obstructive pulmonary disease. Drugs 2003;63(4):379-87. [PubMed: 12558460]

26. Young T, Shahar E, Nieto FJ, Redline S, Newman AB, Gottlieb DJ, et al. Predictors of sleepdisordered breathing in community-dwelling adults: the Sleep Heart Health Study. Arch Intern Med 2002;162(8):893-900. [PubMed: 11966340]

27. Ohayon MM, Carskadon MA, Guilleminault C, Vitiello MV. Meta-analysis of quantitative sleep parameters from childhood to old age in healthy individuals: developing normative sleep values across the human lifespan. Sleep 2004;27(7):1255-73. [PubMed: 15586779]

28. Buysse D, Reynolds IC, Monk T, Berman S, Kupfer D. The Pittsburgh Sleep Quality Index: A New Instrument for Psychiatric Practice and Research. Psychiatry Research 1989;28:193-213. [PubMed: 2748771]

29. Dolberg OT, Hirschmann S, Grunhaus L. Melatonin for the treatment of sleep disturbances in major depressive disorder. Am J Psychiatry 1998;155(8):1119-21. [PubMed: 9699707]

30. Gentili A, Weiner D, Kuchibhatla M, Edinger J. Test-retest reliability of the Pittsburgh sleep quality index in nursing home residents [letter]. J Am Geriatr Soc 1995;43(11):1317-8. [PubMed: 7594173]

31. Beck SL, Schwartz AL, Towsley G, Dudley W, Barsevick A. Psychometric evaluation of the Pittsburgh Sleep Quality Index in cancer patients. J Pain Symptom Manage 2004;27(2):140-8. [PubMed: 15157038]

32. Carter PA. Caregivers' descriptions of sleep changes and depressive symptoms. Oncol Nurs Forum 2002;29(9):1277-83. [PubMed: 12370697]

33. Sarna L, Brecht ML. Dimensions of symptom distress in women with advanced lung cancer: a factor analysis. Heart Lung 1997;26(1):23-30. [PubMed: 9013218]

34. Chochinov HM, Wilson KG, Enns M, Lander S. “Are you depressed?” Screening for depression in the terminally ill. Am J Psychiatry 1997;154(5):674-6. [PubMed: 9137124]

35. Sloan JA, Loprinzi CL, Kuross SA, Miser AW, O'Fallon JR, Mahoney MR, et al. Randomized comparison of four tools measuring overall quality of life in patients with advanced cancer. J Clin Oncol 1998;16(11):3662-73. [PubMed: 9817289] 
36. Gough IR, Furnival CM, Schilder L, Grove W. Assessment of the quality of life of patients with advanced cancer. Eur J Cancer Clin Oncol 1983;19(8):1161-5. [PubMed: 6684561]

37. Baker F, Denniston M, Smith T, West MM. Adult cancer survivors: How are they faring? Cancer. 2005

38. Foley D, Ancoli-Israel S, Britz P, Walsh J. Sleep disturbances and chronic disease in older adults: results of the 2003 National Sleep Foundation Sleep in America Survey. J Psychosom Res 2004;56 (5):497-502. [PubMed: 15172205]

39. Sarna L, Padilla G, Holmes C, Tashkin D, Brecht ML, Evangelista L. Quality of life of long-term survivors of non-small-cell lung cancer. J Clin Oncol 2002;20(13):2920-9. [PubMed: 12089220]

40. Passik SD, Whitcomb LA, Kirsh KL, Theobald DE. An unsuccessful attempt to develop a singleitem screen for insomnia in cancer patients. J Pain Symptom Manage 2003;25(3):284-7. [PubMed: 12614963]

41. Stewart DE, Wong F, Duff S, Melancon CH, Cheung AM. "What doesn't kill you makes you stronger": an ovarian cancer survivor survey. Gynecol Oncol 2001;83(3):537-42. [PubMed: 11733968]

42. Sarna L, Evangelista L, Tashkin D, Padilla G, Holmes C, Brecht ML, et al. Impact of respiratory symptoms and pulmonary function on quality of life of long-term survivors of non-small cell lung cancer. Chest 2004;125(2):439-45. [PubMed: 14769722]

43. Katz DA, McHorney CA. The relationship between insomnia and health-related quality of life in patients with chronic illness. J Fam Pract 2002;51(3):229-35. [PubMed: 11978233]

44. Davidson JR, Waisberg JL, Brundage MD, MacLean AW. Nonpharmacologic group treatment of insomnia: a preliminary study with cancer survivors. Psychooncology 2001;10(5):389-97. [PubMed: 11536417]

45. Jacobs GD, Pace-Schott EF, Stickgold R, Otto MW. Cognitive behavior therapy and pharmacotherapy for insomnia: a randomized controlled trial and direct comparison. Arch Intern Med 2004;164(17): 1888-96. [PubMed: 15451764]

46. Krystal AD, Walsh JK, Laska E, Caron J, Amato DA, Wessel TC, et al. Sustained efficacy of eszopiclone over 6 months of nightly treatment: results of a randomized, double-blind, placebocontrolled study in adults with chronic insomnia. Sleep 2003;26(7):793-9. [PubMed: 14655910]

47. Roth T, Stubbs C, Walsh JK. Ramelteon (TAK-375), a selective MT1/MT2-receptor agonist, reduces latency to persistent sleep in a model of transient insomnia related to a novel sleep environment. Sleep 2005;28(3):303-7. [PubMed: 16173650] 


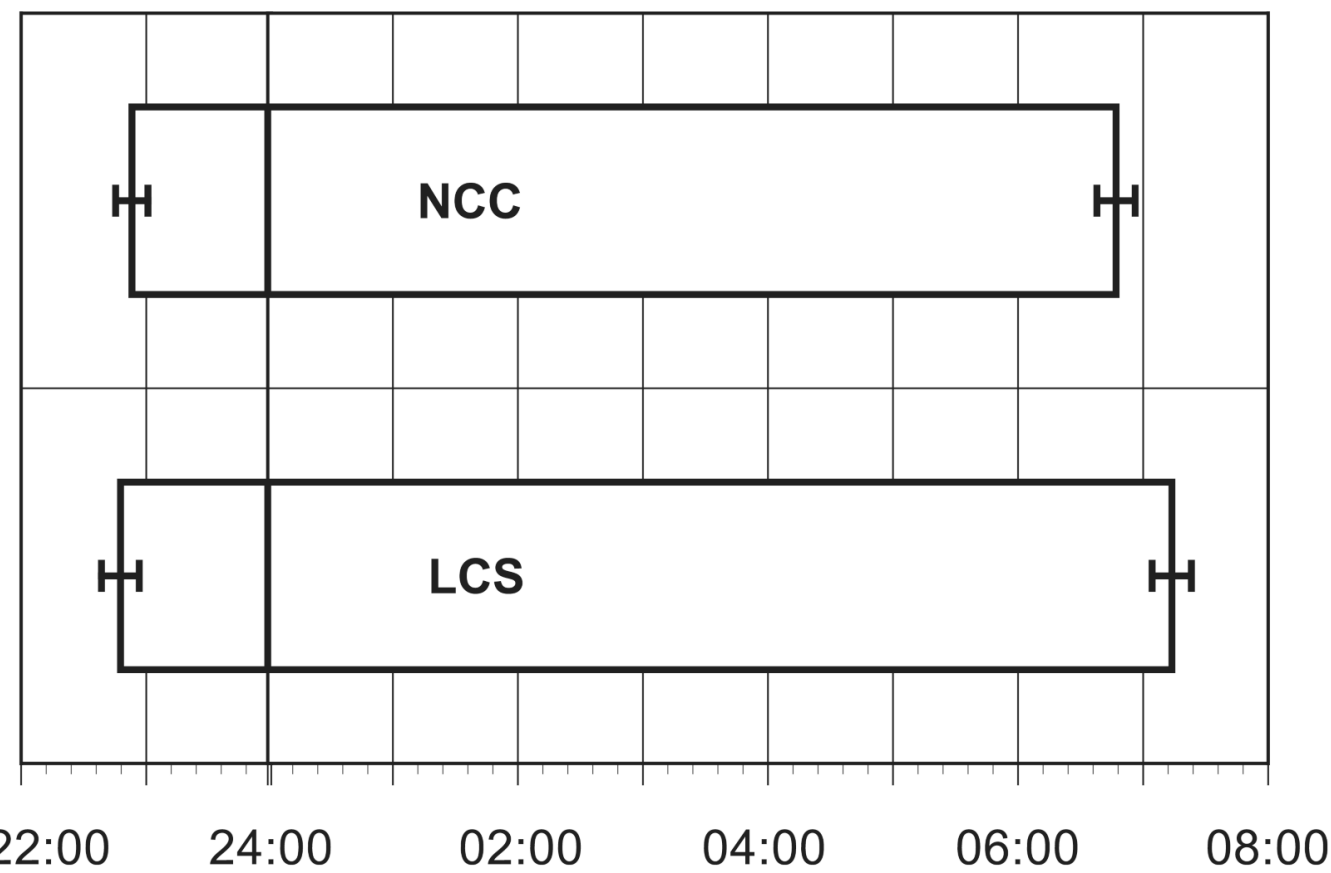

Figure 1.

Mean time subjects went to bed and mean time subjects got out of bed for lung cancer survivors (LCS) and non-cancer controls (NCC). 


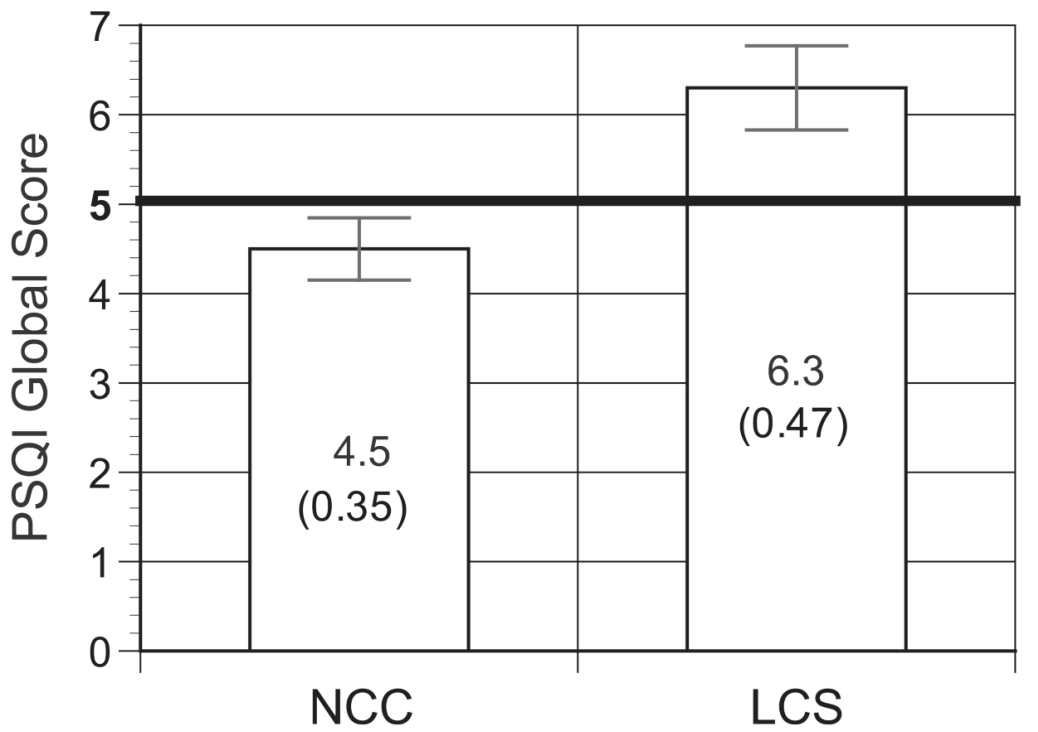

Figure 2.

Bar graph showing global Pittsburgh Sleep Quality Index values between lung cancer survivors (LCS) and non-cancer controls (NCC). Values above 5 (indicated by the heavy black line) suggest poor sleep. 


\section{Table 1}

Demographic characteristics of study population

\begin{tabular}{lcc}
\hline Variable & $\begin{array}{c}\text { Lung Cancer Survivors } \\
(\mathbf{N}=\mathbf{7 6})\end{array}$ & $\begin{array}{c}\text { Non-Cancer Controls } \\
(\mathbf{N = 7 8 )}\end{array}$ \\
\hline Age (year) & $73.6+1-6.7$ & $74.8+/-6.5$ \\
(range: $64-92)$ & \\
Gender & $($ range: $61-89)$ & $30(38.5 \%)$ \\
Male & $29(38.2 \%)$ & $48(61.5 \%)$ \\
Female & $47(61.8 \%)$ & $4(5 \%)$ \\
Race & $10(13 \%)$ & $74(95 \%)$ \\
African-American & $66(87 \%)$ & \\
Caucasian & & \\
\hline
\end{tabular}


Table 2

Common causes of difficulty sleep cited by lung cancer survivors and non-cancer controls

\begin{tabular}{lccc}
\hline Symptom & $\begin{array}{c}\text { Lung Cancer Survivors } \\
(\mathbf{N = 7 6 )}\end{array}$ & $\begin{array}{c}\text { Non-Cancer Controls } \\
(\mathbf{N = 7 8 )})\end{array}$ & p-value (Chi-square test) \\
\hline Pain & $11.8 \%$ & $24.4 \%$ & $0.4 \%$ \\
Dyspnea & $15.8 \%$ & $61.5 \%$ & 0.04 \\
Nocturia & $75.0 \%$ & $12.8 \%$ & 0.06 \\
Too hot & $18.4 \%$ & $4.1 \%$ & 0.07 \\
Too cold & $7.4 \%$ & $5.1 \%$ & 0.3 \\
Bad dreams & $7.9 \%$ & $23.1 \%$ & 0.5 \\
Other problems & $28.6 \%$ & $7.7 \%$ & 0.4 \\
Coughing or snoring loudly & $9.2 \%$ & 0.7 \\
\hline
\end{tabular}


Table 3

Univariate and multivariate regression results for possible determinants of quality of life.

\begin{tabular}{lccc}
\hline Variable & Univariate Statistical Comparison & Multivariate Regression Coefficients \\
\cline { 3 - 3 } & & Parameter Coefficient & Standardized Parameter Coefficient \\
\hline value
\end{tabular}

